\title{
РЕЗУЛЬТАТЫ ЭКСПЕРИМЕНТАЛЬНЫХ ИССЛЕДОВАНИЙ ГИДРОИМПУЛЬСНОГО МЕХАНИЗМА ДЛЯ БУРЕНИЯ ПИЛОТНЫХ СКВАЖИН ПРИ ПРОКЛАДКЕ ТРУБОПРОВОДОВ
}

\author{
Саруев Лев Алексеевич 1 , \\ saruevla@tpu.ru
}

\author{
Шадрина Анастасия Викторовна ${ }^{1}$, \\ kr_nas_sh@tpu.ru
}
Мельнов Кирилл Вячеславович1, kvm11@tpu.ru

\author{
Саруев Алексей Львович 1 , \\ saruev@tpu.ru \\ 1 Национальный исследовательский Томский политехнический университет, \\ Россия, 634050, г. Томск, пр. Ленина, 30.
}

\begin{abstract}
Актуальность. В горном деле и в других отраслях промышленности широко применяются гидравлические ударные механизмы, которые в настоящее время продолжают активно совершенствоваться. В частности, появляются публикации по возможности управления амплитудой и длительностью формируемых гидромеханическими системами импульсов давления 8 замкнутых камерах, через которые поршни-ударники передают энергию удара буровому породоразрушающему инструменту. При этом увеличивается длительность силовых импульсов, что существенно повышает эфрфективность использования их энергии на разрушение грунта, а также сглаживаются чрезмерные динамические нагрузки на элементы бурового инструмента. Однако в таких ударных механизмах остаются поршни-ударники, в процессе работы которых происходят основные динамические потери энергии, связанные с возвратно-поступательными движениями бойков в гидроцилиндре ударного механизма. Был разработан принципиально новый безбойковый гидроимпульсный механизм, который в зависимости от твердости разрушаемой среды регулирует амплитуду и длительность фоормируемых им силовых импульсов в автоматическом режиме без привлечения каких-либо средств управления. Однако для научного обоснования взаимосвязи его основных параметров и работоспособности в целом необходимо провести специальные экспериментальные исследования, результаты которых позволили бы рекомендовать гидроимпульсный механизм для бурения пилотных скважин при прокладке трубопроводов, что, безусловно, является актуальной научно-технической задачей.
\end{abstract}

Цель: обоснование и экспериментальная проверка в лабораторных условиях работы физической модели принципиально нового гидроимпульсного механизма для интенсификации процесса разрушения горных пород или грунта с возможными включениями повышенной твёрдости при бурении пилотных скважин с бестраншейной прокладкой трубопроводов.

объектом данного исследования является безбойковый гидроимпульсный механизм с замкнутьм объёмом жидкости, в котором формируются силовые импульсы, повышающие эффрективность разрушения грунта и горных пород с включениями повышенной твердости за счет автоматического управления их амплитудой и длительностью.

Предмет: закономерности и взаимосвязи основных кинематических и динамических параметров фризической модели гидроимпульсного механизма с иелью оценки его работоспособности и преимуществ перед другими ударными механизмами, включающими замкнутый объём жидкости в качестве промежуточного тела, передающего ударнье импульсы в буровой инструмент.

Методы: анализ научно-технической информации по повышению производительности бурильных машин ударного действия; моделирование динамических процессов гидроимпульсного механизма и проведение экспериментальных исследований его физической модели для выявления взаимосвязей его основных параметров и работоспособности; сравнение разработанного гидроимпульсного механизма с известными гидроударными механизмами, использующими возможности управления амплитудой и длительностью ударных импульсов при передаче их энергии через замкнутый объём жидкости и бурильную колонну на породразрушающий инструмент для интенсификации процесса разрушения горных пород повышенной твердости.

Результаты. Дана оценка работоспособности гидромеханического механизма и его преимущества по сравнению с существующими гидроударными механизмами. Поскольку в предлагаемом гидроимпульсном механизме нет бойка, в нем отсутствуют основные динамические потери энергии, которые имеют место при возвратно-поступательном движении поршнябойка в гидроцилиндре. Кроме того, благодаря возможности работы гидроимпульсного механизма без маслостанции, отсутствуют потери энергии на перекачивание жидкости. Коэфффициент полезного действия гидроимпульсного механизма выше, чем у других существующих гидроударных систем ещё и потому, что он формирует силовые импульсы с частотным спектром, в котором практически отсутствуют высокочастотные составляющие, не способствующие интенсификации процесса разрушения горных пород, при этом увеличивается доля энергии силовых импульсов в продольных волнах деформации, что обеспечивает повышение механической скорости бурения скважин. Установлено, что гидроимпульсный механизм автоматически регулирует амплитуду и длительность силовых импульсов в зависимости от твердости разрушаемой среды и не требует дополнительной системы управления этим процессом.

Ключевые слова:

Силовой импульс, рукав высокого давления, гидроимпульсный механизм, система с замкнутым контуром, импульсы давления, амплитуда и длительность импульсов, бестраншейная прокладка трубопроводов. 


\section{Введение}

Бестраншейная прокладка трубопроводов обычно предусматривает бурение пилотных скважин. При этом нередко встречаются горные породы с перемежающейся твердостью, для эффективного бурения которых рационально применять установки, оснащенные ударными механизмами [1-3]. В результате нанесения ударов по торцу хвостовика бурильной колонны в последней формируются волны деформации сжатия, которые, распространяясь со скоростью звука к породоразрушающему инструменту, воздействуют на него в виде силовых импульсов, обеспечивая эффективное разрушение горных пород. В то же время силовые импульсы создают чрезмерные динамические нагрузки на буровой инструмент. Известно, что формируемые напряжения в результате ударов бойка прямо пропорциональны его предударной скорости и допускаемая величина её составляет не более 10-12 м/с Эти ограничения скорости бойка вынуждают разработчиков ударных механизмов повышать энергию ударов за счёт увеличения массы бойков, что приводит к увеличению габаритов и массы всего вращательно-ударного механизма бурильных установок [4-11]. Для решения проблемы повышения энергии удара путём увеличения предударной скорости бойка фирмой Джой (США) был создан молот, в котором передача энергии удара бойка на буровой инструмент осуществлялась через замкнутый столбик жидкости [12]. Учитывая положительный опыт передачи энергии удара через промежуточный элемент в виде замкнутого столбика жидкости, в настоящее время активно продолжаются исследования, связанные с разработкой и совершенствованием ударных систем с жидкостными камерами [13-15]. Авторы этих исследований убедительно доказывают целесообразность повышения таким образом эффективности разрушения горных пород благодаря возможности управления амплитудой и длительностью формируемых силовых импульсов.

Увеличенная длительность силовых импульсов существенно повышает эффективность использования их энергии на разрушение грунта, а также сглаживаются чрезмерные динамические нагрузки на элементы бурового инструмента. Однако в таких ударных механизмах остаются поршни-ударники, в процессе работы которых происходят основные динамические потери энергии, связанные с возвратнопоступательными движениями бойков в гидроцилиндре ударного механизма.

В Томском политехническом университете (ТПУ) разработан принципиально новый безбойковый гидроимпульсный механизм, который в зависимости от твердости разрушаемой среды регулирует амплитуду и длительность формируемых им силовых импульсов в автоматическом режиме без привлечения какихлибо средств управления. Новизна и оригинальность гидроимпульсного механизма подтверждена патентами на полезную модель [16, 17].

Исследования профессора А.С. Сердечного показали [18], что даже при передаче удара через замкну- тый столбик жидкости формируются силовые импульсы со сложным частотным спектром, в котором значительную долю представляют высокочастотные составляющие (поперечные волны), скорость распространения которых по бурильной колонне в 1,5 раза ниже скорости продольных волн деформации, и, дойдя до породоразрушающего инструмента, поперечные волны не оказывают влияния на внедрение его в обрабатываемую среду, то есть не совершают полезной работы при бурении скважин.

\section{Отличительная особенность предложенного} гидроимпульсного механизма

Отличительной особенностью разработанного в ТПУ гидроимпульсного механизма является отсутствие в нём бойка (ударника). Принципиальная схема работы такого механизма формирования силовых импульсов представлена на рис. 1 , где $F_{\text {ос }}-$ усилие, создаваемое податчиком бурильной установки.

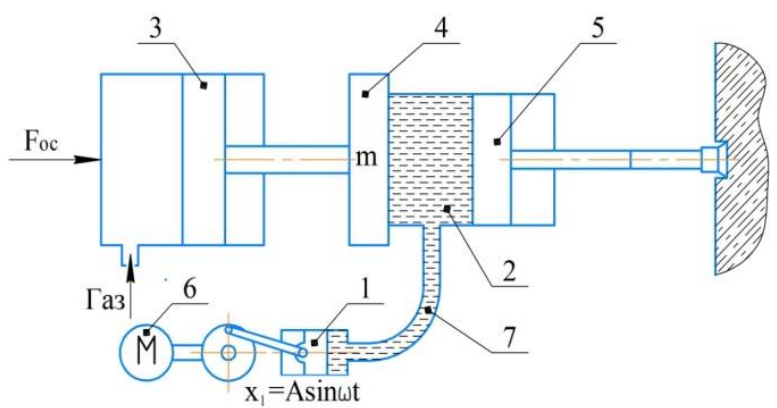

Pис. 1. Обобщенная схема работы гидромеханической системы: 1 - гидропульсатор; 2 - жидкость в замкнутой системе; 3 - поршень пневмоцилиндра, обеспечивающий упругое поджатие активной инериионной массы; 4 - инериионная масса m с корпусом силового ичилиндра; 5 - поршень, связанный с хвостовиком бурильной колонны и формирующий силовые импульсы, которые распространяются по ней к породоразрушаюшему инструменту; 6 - электродвигатель гидропульсатора; 7 - рукав высокого давления (РВД)

Fig. 1. Schematic view of the hydromechanical system operation: 1 -pulsator; 2 -liquid in a closed-loop system; 3 -plunger air feeder; 4 -inertial mass $\mathrm{m}$ with the power cylinder; 5 - plunger connected to the drill string shank and forming power pulses that propagate along it to the rock drill tool; 6 -electric motor of pulsator; 7 - high-pressure hose

Упругие характеристики рукавов высокого давления представлены на рис. 2. Они свидетельствуют о нелинейности зависимости давления, создаваемого в них, от объема закачиваемой жидкости.

Импульсы давления формируются в гидроцилиндре в результате взаимодействия колебания давления жидкости в замкнутой полости гидросистемы механизма, создаваемого гидропульсатором, а также колебания инерционной активной массы, жестко связаной с корпусом гидроцилиндра (рис. 1). Формирование импульсов давления жидкости в гидросистеме, включая давление в силовом гидроцилиндре, осуществляется следующим образом. 


\section{$P$, МПа}

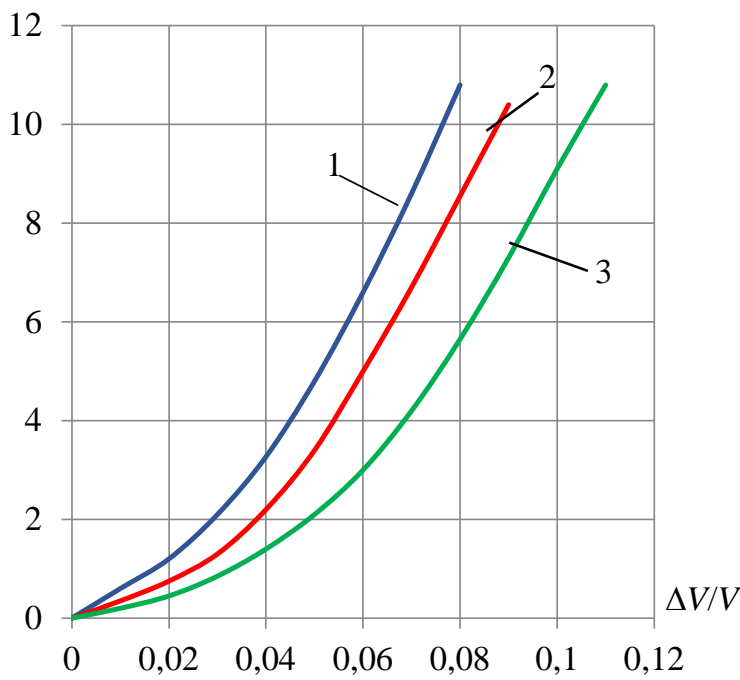

Рис. 2. Упругие характеристики рукавов высокого давления, имеющих внутренний диаметр 20 мм различной длины и объёма: $1-L=0,95 \mathrm{M} ; V=298 \mathrm{~cm}^{3}$; $2-L=1,16 \mu ; V=364 \mathrm{~cm}^{3} ; 3-L=1,45 \mathrm{M} ; V=455$ $c M^{3}$

Fig. 2. Elastic characteristics of high-pressure hoses with inner diameter $20 \mathrm{~mm}$ and various lengths and volumes: $1-L=0,95 \mathrm{~m} ; V=298 \mathrm{~cm}^{3} ; 2-L=1,16 \mathrm{~m}$; $V=364 \mathrm{~cm}^{3} ; 3-L=1,45 \mathrm{~m} ; V=455 \mathrm{~cm}^{3}$

Плунжер вытесняет из полости гидропульсатора жидкость, которая повышает давление во всем замкнутом объеме гидросистемы на величину $\Delta P=$ $f\left(\frac{\Delta V}{V}\right)$, где $\Delta V$ - изменение объёма жидкости в замкнутом объёме гидросистемы гидроимпульсного механизма (рис. 1). Поршень - 3 пневмоцилиндра, поджатый давлением воздуха, работает как пневмо- пружина, жесткость которой существенно меньше жесткости замкнутого объёма жидкости гидросистемы. В этих условиях при работе гидропульсатора происходит раскачка активной инерционной массы $m$ с корпусом гидроцилиндра и сдвиг по фазе колебаний массы $m$ по отношению к вынужденным движениям плунжера гидропульсатора. Причём при одновременной работе двух колебательных контуров в режиме близком к резонансному движение массы $m$ в сторону хвостовика бурильной колонны происходит во время движения плунжера на вытеснение жидкости из полости гидропульсатора объёмом $\Delta V$ в замкнутую полость гидросистемы, которую можно рассматривать как гидропружину. Рукав высокого давления, входящий в состав гидросистемы гидроимпульсного механизма, обладает нелинейной характеристикой изменения давления от объёма вытесненной в него жидкости из гидромеханической системы. В силовом гидроцилиндре происходят изменения давления, воздействующие на поршень - 5 гидроцилиндра, который, в свою очередь, создает силовые импульсы в виде волн деформации сжатия в бурильной колонне. Последние распространяются по ней на забой скважины, обеспечивая интенсификацию разрушения встречающихся включений повышенной твердости. Это позволяет увеличить механическую скорость бурения (рис. 1). При этом предварительно давление в силовом гидроцилиндре создается податчиком бурильной установки. Как показали экспериментальные исследования, в данной гидромеханической системе силовые импульсы формируются только в направлении на забой скважины, и бурильная установка при устойчивой работе гидроимпульсного механизма защищена им от силовых импульсных воздействий (рис. 3).

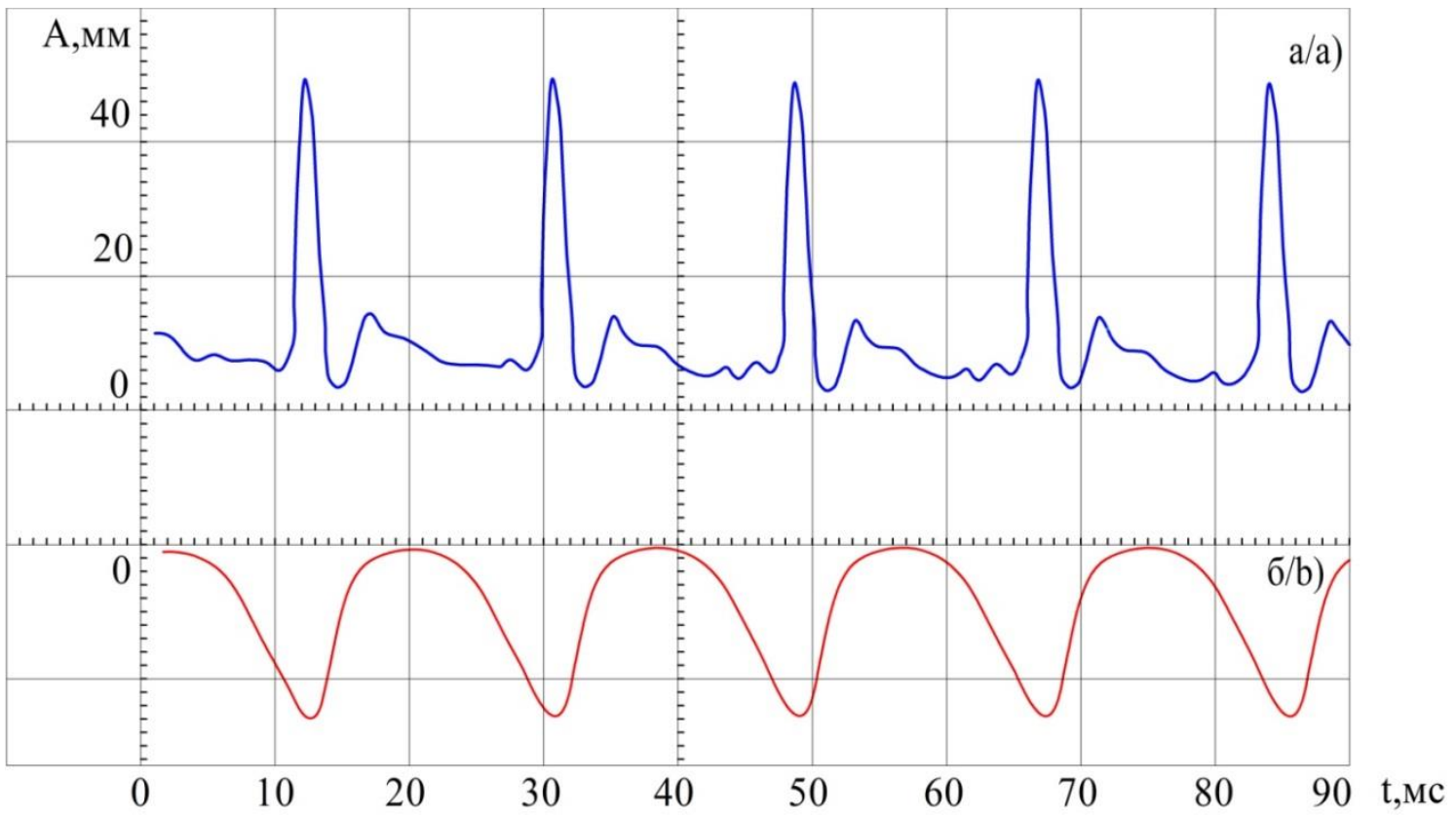

Рис. 3. Осииллограммы: а) силовые импульсы в колонне труб; б) характер линейных перемещений корпуса силового цилиндра, жестко связанного с инериионной массой

Fig. 3. Oscillograms: a) power impulsions in the drill rod; b) movement of the active mass of the hydropulse mechanism 


\section{Методика и результаты}

\section{экспериментальных исследований}

Для проведения исследований гидроимпульсного механизма была разработана и изготовлена экспериментальная установка, общий вид и основные элементы которой приведены на рис. 4.

Три рукава высокого давления с длиной от 0,67 до 4,5 м были приняты в эксперименте, при этом их диаметры составили 12, 16 и 20 мм. Инерционная масса установки принимала значения от 30 до 270 кг. Осевое усилие подачи использовалось для оценки влияния создания предварительного давления жидкости в гидросистеме гидроимпульсного механизма на нелинейные характеристики различных РВД и задавалась в пределах от нуля до 12 кН. Вынужденные частоты гидропульсатора в эксперименте составляли 25 и 50 Гц (без учета величины скольжения ротора электродвигателя).

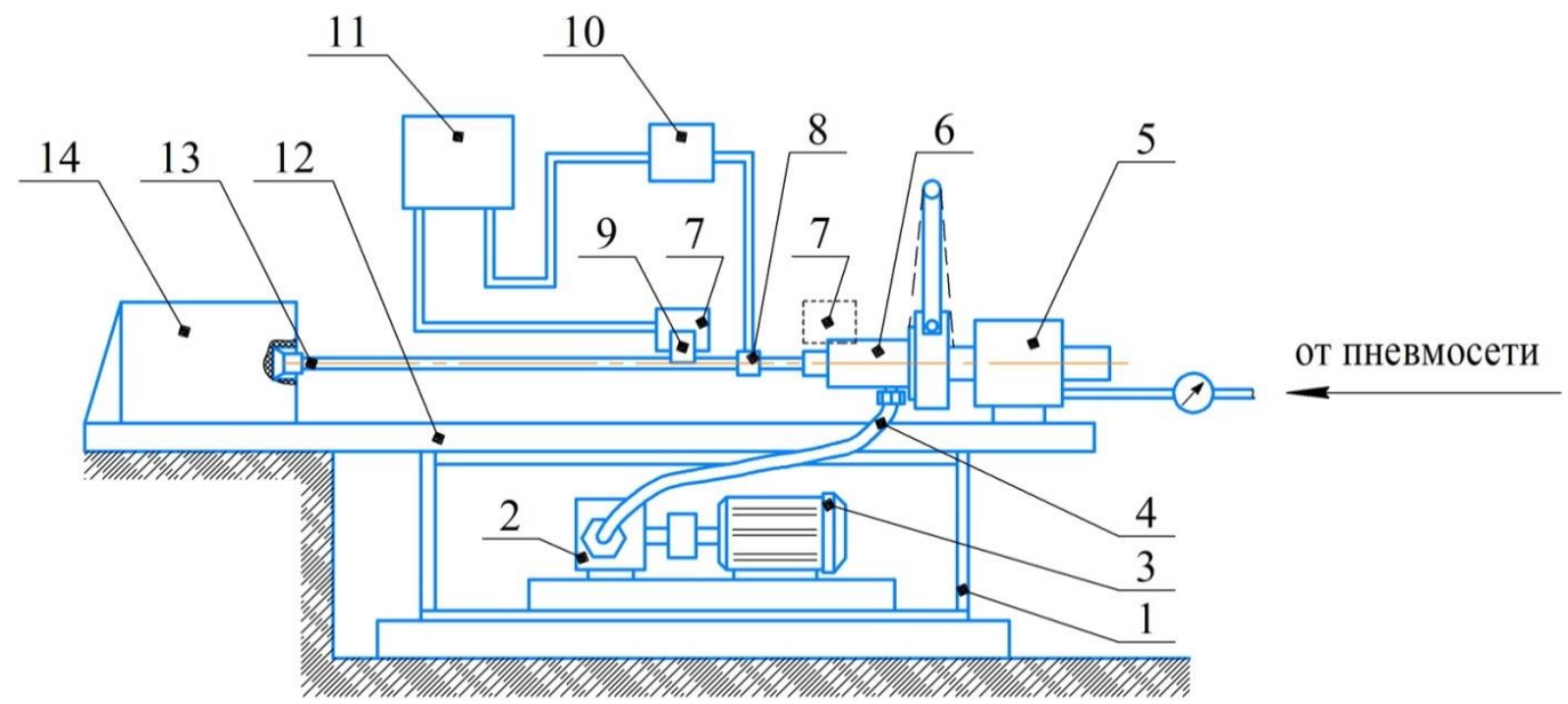

Pис. 4. Лабораторная установка для исследования гидроимпульсного силового механизма: 1 - сварная несущая рама; 2 - пульсатор; 3 - электродвигатель; 4 - рукав высокого давления; 5 - пневмоподатчик, обеспечивающий постоянное поджатие активной инериионной массы; 6 - силовой гидрочилиндр; 7 - датчики линейных перемещений; 8 - тензодатчики сопротивления для записи силовых импульсов в бурильной трубе; 9 - шторка датчика линейных перемещений, жестко связанная с бурильной колонной; 10 - усилитель постоянного тока; 11 - осииллограф; 12 - направляющая рама; 13 - бурильная труба с породоразрушающим инструментом; 14 - горная порода

Fig. 4. Test bench for the study of hydraulic mechanism: 1 - bed frame; 2 - pulsator; 3 - pulsator's motor; 4 - high pressure hose; 5 - pneumatic feeder; 6 - hydraulic power cylinder; 7 -linear displacement transducer; 8 -resistive strain gages; 9 - curtain of the linear displacement sensor, rigidly connected with the drill pipe; 10 - DC amplifier; 11 - oscilloscope; 12 - guide frame; 13 -drill pipe; 14 - rock

Увеличение амплитуды и энергии силовых импульсов в бурильной колонне наблюдается при уменьшении длины РВД и увеличении его диаметра (рис. 5).

Эксперимент показал, что при постоянном значении объёма жидкости $\Delta \mathrm{V}$, вытесняемом плунжером пульсатора в гидросистему гидроимпульсного механизма, чем меньше отношение этого объёма к общему замкнутому объёму гидросистемы, тем меньше амплитудное значение давления в гидросистеме, в том числе в силовом гидроцилиндре. При уменьшении диаметра РВД в нём повышается сопротивление пульсирующим движениям жидкости, что оказывает негативное влияние на процесс формирования силовых импульсов, снижая их амплитуду и энергию. Исследования показали, что осевое усилие подачи, воздействуя на пневмопружину и через неё на инерционную массу $m$, закреплённую на силовом гидроцилиндре, создаёт в последнем предварительное начальное давление, составляющее во время экспериментов в среднем 3 МПа. В эксперименте отмечалась нелинейная зависимость давления в силовом гидроцилиндре от величины вытесняемой плунжером пульсатора объёма жидкости $\Delta V$ в общий замкнутый объём $V$ гидросистемы, который остаётся постоянной величиной. Установлено, что чем выше осевое усилие подачи, тем эффективнее формируется амплитуда силовых импульсов в бурильной колонне.

Осциллограммы на рис. 6 показывают, что время между двумя соседними силовыми импульсами составляет 40 мс, то есть частота колебательного контура с инерционной массой $m$ составит 25 Гц, что в 2 раза ниже вынужденной частоты гидропульсатора. Согласно теории колебаний [19] в данном случае этот контур становится захватывающим парциальную частоту автоколебательной системы и благодаря снижению частоты формируемых силовых импульсов позволяет повышать их амплитуду и энергию. Вместе с тем, как следует из представленных на рис. 7 зависимостей импульсной силы от усилия подачи, на амплитуду импульса существенно влияет инерционная масса $m$. Амплитуда силовых импульсов растет с увеличением активной инерционной массы (рис. 7). 

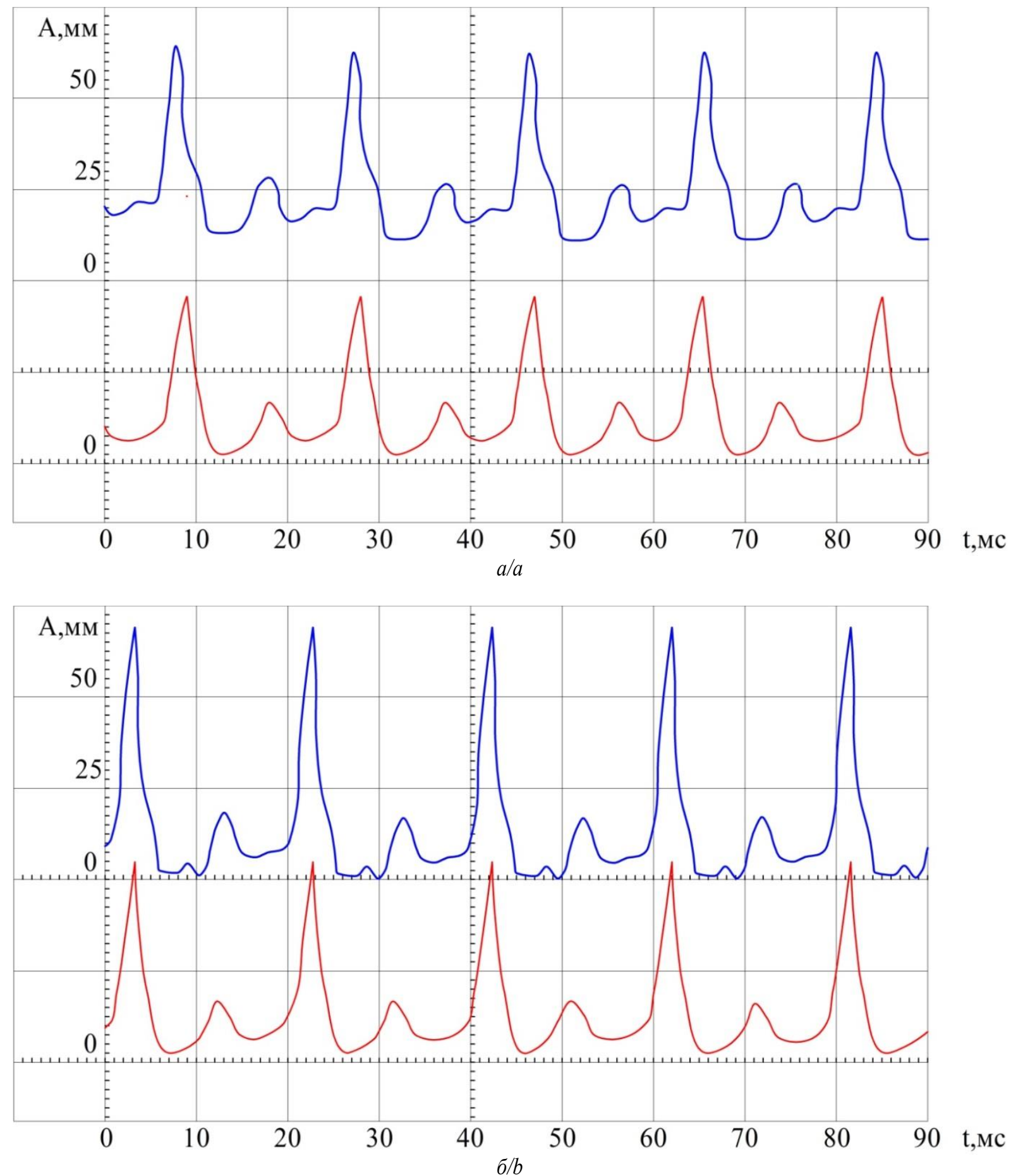

Рис. 5. Формируемые силовые импульсы: верхние осииллограммы - усилие в бурильной трубе; нижние осциллограммы - давление в гидроцилиндре: а) диаметр РВД 16 мм, длина РВД 2,5 м; б) диаметр РВД 20 мм, длина РВД 0,95 м. Вынужденная частота гидропульсатора 25 Ги (развертка осииллографа 25 мс/см экрана)

Fig. 5. Power pulses: upper oscillograms - axial load in the drill pipe; lower oscillograms - pressure in the hydraulic cylinder: a) high-pressure hose diameter $16 \mathrm{~mm}$, high-pressure hose length 2,5 $\mathrm{m}$; b) high-pressure hose diameter $20 \mathrm{~mm}$, high-pressure hoses length 0,95 m. Forcing frequency of the hydraulic pulsator $25 \mathrm{~Hz}$ (oscilloscope sweep $25 \mathrm{~ms} / \mathrm{cm}$ of screen) 

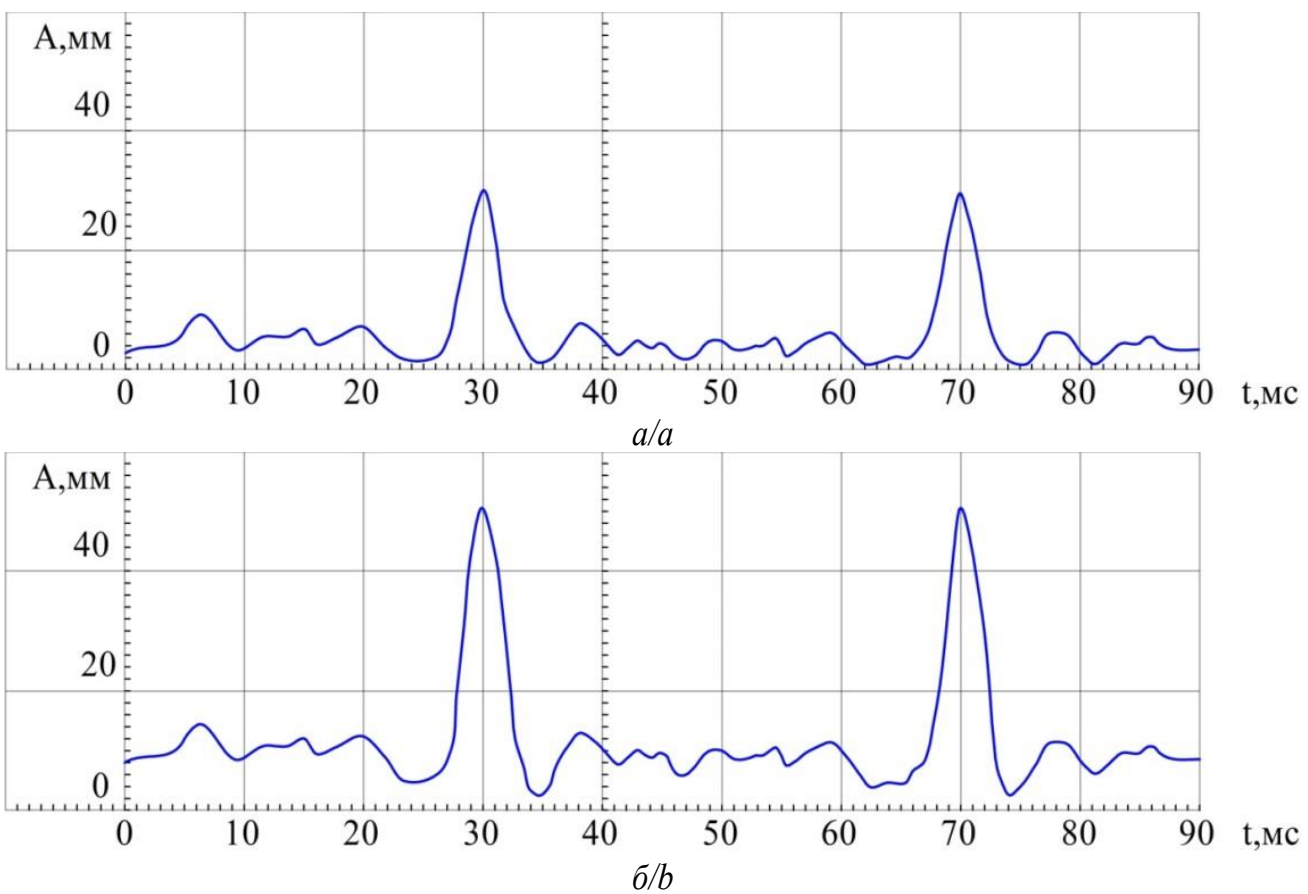

Рис. 6. Осииллограммы импульсов, формируемых в буровой колонне при разном значении осевого усилия податчика буровой установки: а) 9,4 кН; б) 11,75 кН; диаметр РВД 20 мм, длина РВД 1,16 м. Частота вынужденных колебаний гидропульсатора $\omega_{2}=50$ Ги; масштаб $\mu_{t}=10$ мс/см экрана осииллографа

Fig. 6. Oscillograms of pulses generated in the drill string at different values of the axial load: a) 9,4 kN; b) 11,75 kN; highpressure hose diameter $20 \mathrm{~mm}$, high-pressure hose length 1,16 m. Forcing frequency of the hydraulic pulsator $50 \mathrm{~Hz}$; scale $\mu \mathrm{t}=10 \mathrm{~ms} / \mathrm{cm}$ of the oscilloscope screen

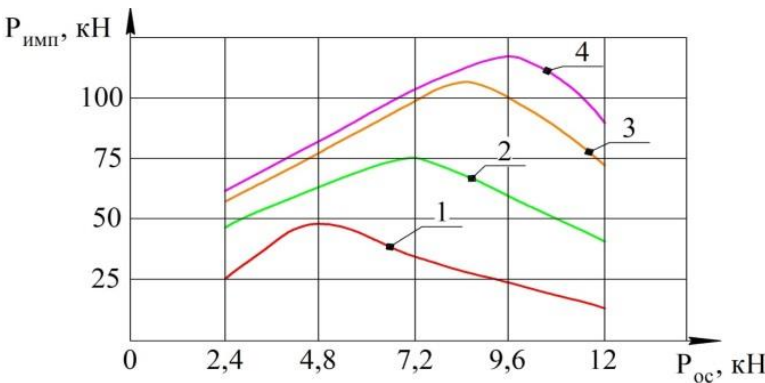

Рис. 7. Характеристика, показывающая влияние усилия подачи и активной инерционной массы на амплитуду силовых импульсов в колонне труб при объёме рукава высокого давления: $V=455 \mathrm{~cm}^{3}$ $(d=20 \mathrm{M \mu}, L=1,45 \mathrm{~m}): 1-m=30 \kappa 2 ; 2-m=70 \kappa 2$; $3-m=110 \kappa 2 ; 4-m=230 \kappa 2$

Fig. 7. Characteristic showing the influence of the axial load and inertial mass on the amplitude of the power pulses in the drill string at the volume of the high-pressure hose: $V=455 \mathrm{~cm}^{3}(d=20 \mathrm{~mm}, L=1,45 \mathrm{~m}): 1-m=30 \mathrm{~kg}$; $2-m=70 \mathrm{~kg} ; 3-m=110 \mathrm{~kg} ; 4-m=230 \mathrm{~kg}$

Выявлено, что представленная система работает устойчиво со сдвигом фаз колебаний активной массы и вынужденных колебаний плунжера пульсатора в диапазоне от 120 до 150 градусов. Таким образом, варьируя частоту колебаний плунжера и величину активной массы $m$, можно определить требуемую жесткость отдельных элементов системы. В частности, можно определить жесткости гидро- и пневмопружины гидроимпульсного механизма, что позволит формировать силовые импульсы с параметрами, необходимыми для эффективного разрушения твердых включений, кото- рые могут встречаться в процессе бурения пилотных скважин, а также благодаря пологому переднему фронту снизить уровень динамических нагрузок на резьбовые соединения бурильных колонн, повышая надежность их работы. Кроме того, ряд исследователей отмечают, что силовые импульсы с пологим передним фронтом в большей степени используют свою энергию на разрушение горной породы [20].

Взаимосвязь энергии силовых импульсов и осевого усилия подачи представлены на рис. 8 .

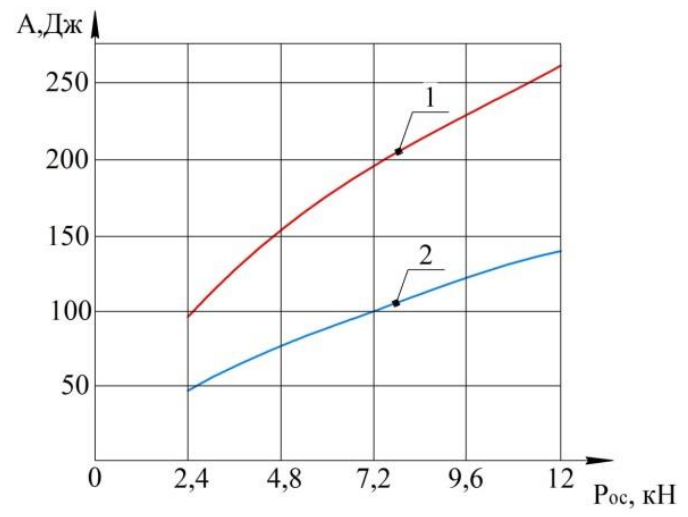

Рис. 8. Взаимосвязь энергии силовых импульсов и осевого усилия подачи: 1 - объём РВД $V=298 \mathrm{~cm}^{3}$, $d=20$ мм; 2 - объём РВД $V=515 \mathrm{~cm}^{3}, d=20$ мм; инерционная масса $m=230$ кг

Fig. 8. Relationship between the energy of power pulses and the axial load: 1 - high-pressure hose volume $V=298 \mathrm{sm}^{3}, d=20 \mathrm{~mm} ; 2$ - high-pressure hose volume $V=515 \mathrm{sm}^{3}, d=20 \mathrm{~mm}$; inertial mass $m=230 \mathrm{~kg}$ 


\section{Моделирование и анализ динамики гидроимпульсного механизма}

Динамику гидроимпульсного механизма можно анализировать, представив его механической схемой замещения (рис. 9). Схема замещения представляет одномассовую колебательную систему с кинематическим возмущением, где $x_{2}$ - перемещение массы $m$, $C_{1}=f(\Delta x)-$ нелинейная упругость замкнутого объёма жидкости, $C_{2}$ - упругость пневмоподатчика (пневмопружины).
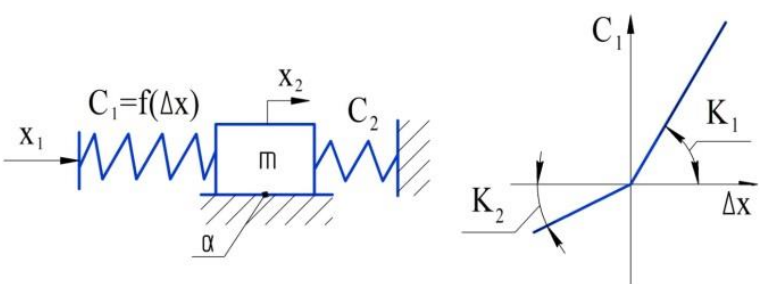

Рис. 9. Механическая схема замещения гидроимпульсного механизма и график нелинейной функиии упругости замкнутого объёма жидкости $C_{l}=f(\Delta x)$

Fig. 9. Mechanical equivalent circuit of the hydraulic pulse mechanism and the graph of the nonlinear elasticity function of a closed-loop volume of liquid $C_{l}=f(\Delta x)$

Для упрощения исследования принимаем поступательное движение системы и перемещение поршня гидроцилиндра равным нулю. Сила, связанная с разрушением породы, заменена вязкой нагрузкой $\alpha \dot{x}_{2}$. Величина $\Delta x=x_{1}-x_{2}$ представляет деформацию нелинейной упругости $C_{1}$. Применяя принцип Даламбера, составляем уравнение движения массы $m$

$$
m \ddot{x}_{2}+\alpha \dot{x}_{2}+\left[C_{1}(\Delta x)+C_{2}\right] x_{2}=C_{1}(\Delta \mathrm{x}) x_{1} .
$$

Из схемы замещения гидроимпульсного механизма (рис. 9) видно, что гидропривод $x_{1}$ имеет обратную отрицательную жёсткую связь по выходной координате $x_{2}$ массы $m$; колебательный контур с массой $\mathrm{m}$ и её координатой $x_{2}$ имеет местную обратную отрицательную связь по скорости и перемещению. При составлении передаточной функции по структурной схеме приходим также к выражению (1), что может служить критерием проверки соответствия гидроимпульсного механизма его механической схеме замещения.

Уравнение (1) позволяет исследовать динамику при различных параметрах гидроимпульсного механизма с учетом получаемых нелинейной зависимости $C_{1}=f(\Delta x)$ и формы периодического входного сигнала $x_{1}$. Амплитудно-частотные характеристики были получены при различных значениях $\kappa_{1}$ нелинейной функции $C_{1}=f(\Delta x)$, а также при различных значениях $b=\frac{\kappa_{2}}{\kappa_{1}}$, изображенных на рис. 11. Зависимости собственных частот $\omega_{0}$ от коэффициента $\sqrt{\mathrm{K}_{1}}$ были получены при $\alpha=0,2$ и $\mathrm{C}_{2}=0,25 \mathrm{C}_{0}$, где $C_{0}-$ упругость рукава высокого давления в статике (рис. 10). Погрешность решения для линейной системы (при $b=1$ ) не превышала двух процентов.

Исследования на модели показали, что теоретический расчет даёт большое расхождение в определе- нии $\omega_{0}$ при уменьшении $b$, начиная с $b=0,7$. Теоретический расчёт проводился по формуле

$$
\omega_{0}=\sqrt{\frac{C_{1} \cdot C_{2}}{m\left(C_{1}+C_{2}\right)}}
$$

где $\mathrm{C}_{1}=\frac{\mathrm{C}_{0}\left(\mathrm{~K}_{1}+b \mathrm{~K}_{2}\right)}{2}$.

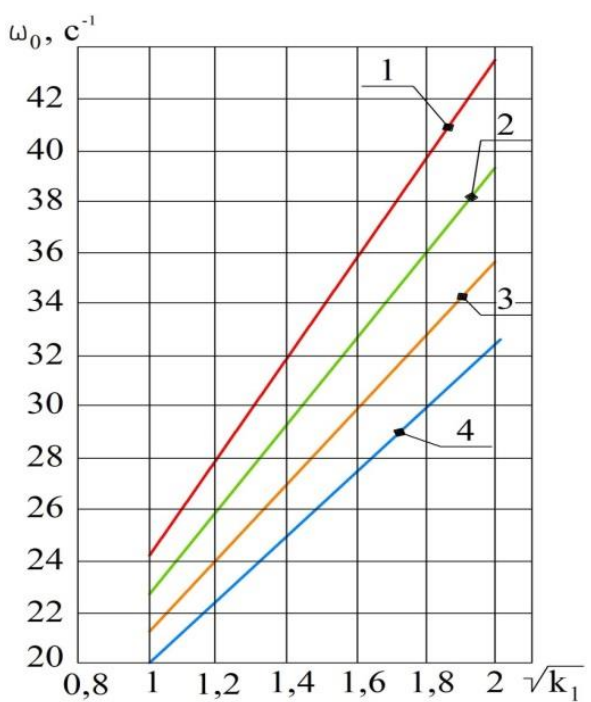

Pис. 10. Зависимость собственных частот $\omega_{0}$ от коэффиичента $\sqrt{\mathrm{K}_{1}}:$ 1) $b=1$; 2) $b=0,7$; 3) $b=0,5$; 4) $b=0,3$, где $b=\frac{\kappa_{2}}{\kappa_{1}}$

Fig. 10. Dependence of eigenfrequency $\omega_{0}$ on the coefficient $\sqrt{\mathrm{K}_{1}}$ : 1) $b=1$; 2) $b=0,7$ 3) $b=0,5$; 4) $b=0,3$, where $b=\frac{\kappa_{2}}{\kappa_{1}}$

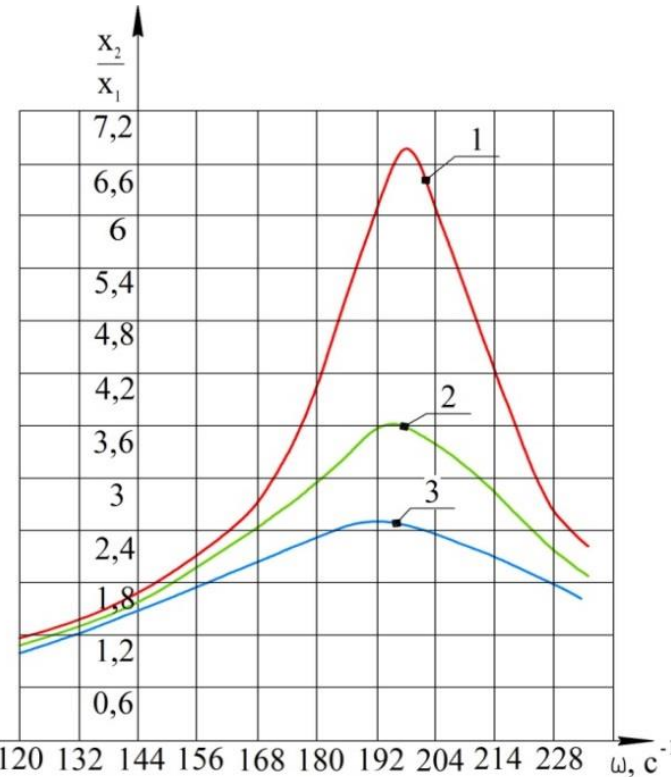

Рис. 11. Алплитудно-частотные характеристики гидромеханической системь: $x_{1}=$ const; $\kappa_{1}=4 ; b=0,3$; 1, 2, 3 -соответственно $\varepsilon=0,1 ; 0,2$ и 0,3

Fig. 11. Amplitude-frequency characteristics of the hydromechanical system: $x_{1}=$ const; $k_{I}=4 ; \quad b=0,3$; for $1,2,3-\varepsilon=0,1 ; 0,2$ and 0,3 respectively 
Установлено, что при $1>b \geq 0,7$ система близка по своим свойствам к линейной и собственная частота может быть рассчитана по формуле (2). При уменьшении $b$, начиная с 0,7 , система проявляет себя как нелинейная, и собственная частота её может быть определена путем математического моделирования.

Поскольку при бурении скважин с использованием гидроимпульсного механизма часть энергии расходуются на разрушение горной породы, были получены амплитудно-частотные характеристики системы при постоянном ходе плунжера гидропульсатора $x_{1}=$ const и $k_{1}=4, b=0,3$. Для такого режима работы гидроимпульсного механизма амплитудно-частотные характеристики представлены на рис. 11 при различной относительной величине расхода энергии системы $\varepsilon$ на разрушение горных пород с перемежающейся твердостью в процессе бурения пилотных скважин.

\section{Заключение}

1. Для обеспечения эффективного режима бурения на участках траектории пилотных скважин с повышенной твердостью разработана и успешно апробирована в лабораторных условиях безбойковая система формирования силовых импульсов, распространяющихся в виде волн упругой деформации по бурильной колонне к породоразрушающему инструменту, позволяющая существенно ускорить проходку указанных интервалов скважины для прокладки трубопроводов.

2. Результаты экспериментальных исследований безбойкового гидроимпульсного механизма позволили выявить взаимосвязи основных параметров и динамические особенности его работы, а также ряд существенных преимуществ при сравнении с другими современными гидроударными механизмами, имеющими замкнутый объем жидкости для передачи через него энергии удара на буровой инструмент.

3. Основным преимуществом гидроимпульсного механизма является повышенный коэффициент полезного действия гидросистемы, в которой отсутствуют динамические потери энергии, связанные с возвратно-поступательными движениями поршнябойка в гидроцилиндрах всех известных гидро-

\section{СПИСОК ЛИТЕРАТУРЫ}

1. Aleksandrova N.I., Kondratenko A.S. Movement of an open-ended pipe with a soil plug under a longitudinal impact // Geotechnical and Geological Engineering. - 2020. - V. 38. - № 4. - P. 3493 3504.

2. Automatic trenchless horizontal directional drilling using quad motors drilling heads / M.A. Kamel, M. Eishafei, A.-W.A. Saif, A.A. Al-Majed // Proc. of 15th International Multi-Conference on Systems, Signals and Devices. - Tunisia-Hammamet, 2018. P. 1172-1177.

3. Analysis and design of pipes installed via direct PIPE technology/ M. Rabiei, K.P. Chang, M. Gelinas, A. Neale // Proc. of Conference: NASTT's 2018. No-Dig Show. - Palm Springs, CA, March 25-29, 2018. - 11 p.

4. Smolyanitskii B.N., Tishenko I.V., Chervov V.V. Improvement prospects for air hammers in building and construction work // Journal of Mining Science. - 2009. - V. 45. - Iss. 4. - P. 363-371. ударных механизмов бурильных установок. Импульсы силы, формируемые механизмом, отличаются более пологим передним фронтом и на порядок большей длительностью импульса по сравнению с обычными ударными механизмами. Это позволяет увеличить внедрение породоразрушающего механизма в грунт и снизить динамические нагрузки на резьбовые соединения труб в бурильной колонне.

4. Формируемые гидроимпульсным механизмом силовые импульсы имеют частотный спектр с пониженной долей высокочастотных составляющих. Основная часть энергии импульсов переносится по бурильной колонне в виде продольных волн деформации, которые представляют низкочастотную составляющую частотного спектра и интенсифицируют процесс разрушения горных пород при бурении скважин.

5. Гидроимпульсный механизм обеспечивает без каких-либо систем или устройств автоматическое регулирование амплитуды силовых импульсов в зависимости от твердости разрушаемой среды. Чем больше твердость горной породы или твердость возможных включений в грунте, тем больше становится жесткость всей гидросистемы замкнутой полости гидроимпульсного механизма, что вызывает увеличение амплитуды формируемых силовых импульсов.

6. В гидроимпульсном механизме силовой гидроцилиндр с поршнем можно заменить сильфоном, что обеспечит герметичность замкнутого объёма жидкости гидросистемы и позволит избежать в таком случае утечек между поршнем и внутренней поверхностью силового гидроцилиндра ввиду отсутствия их в гидросистеме.

Таким образом, анализ результатов проведенных экспериментальных исследований показал возможности эффективного применения гидроимпульсного механизма вместо других известных гидроударных механизмов с передачей энергии удара через замкнутый объём жидкости при бурении пилотных скважин и бестраншейной прокладке трубопроводов в горных породах с перемежающейся твердостью.

5. Effects of working angle on pneumatic down-the-hole hammer drilling / Z. Zhao, Y. Meng, Y. Li, X. Shi, C. Xiang // Rock mechanics and rock engineering . - 2014. - V. 48. - Iss. 5. P. 2141-2155.

6. Gorodilov L.V. Basic properties of one-way action hydraulic percussion system with two piston arresters // Journal of Mining Science. - 2020. - V. 56. - № 6. - P. 105-115.

7. Efimov V.P., Gorodilov L.V. Experimental investigation of shock pulses in the piston-bit system in interaction with rock mass // IOP Conf. Series: Earth and Environmental Science. 2021 - V. 773. URL: $\quad$ https://iopscience.iop.org/article/10.1088/17551315/773/1/012041 (дата обращения: 15.09.2021).

8. Hydraulic-mechanical coupling modeling by bond graph for impact system of a high frequency rock drill drifter with sleele distributor / G. Yonga, L. Deshunb, Ya. Shuyib, L. Xuejuna, Ch. Jianwenc // Automation and construction. - 2016. - V. 63. P. 88-99. 
9. Tishhenko I.V., Chervov V.V., Smoljanickij B.N. Evaluation of layout of air drill hammer with smooth adjustment of impact impulse frequency // Journal of Mining Science. - 2017. - V. 53. № 1. - P. 110-118.

10. Influence of DHT hammer impact energy on drilling-with-casing system performance / V.V. Timonin, S.E. Alekseev, V.N. Karpov, E.M. Chernienkov // Journal of Mining Science. - 2018. - V. 54. № 1. - P. 61-71.

11. Zhukov I.A., Smoljanickij B.N., Timonin V.V. Improvement of down-the-hole air hammer efficiency by optimizing shapes of collding parts // Journal of Mining Science. - 2018. - V. 54. № 2. - P. 37-43.

12. Eremyants V.E., Melis kyzy Nazira About the collision of the rods model choice through the closed liquid volume // Journal of Advanced Research in Technical Science. - 2017. Iss. 6. - P. 11-16.

13. Еремьянц В.Э., Мелис кызы Назира. Модель ударной системы с жидкостной камерой для разрушения прочных и мерзлых грунтов // Автоматизированное проектирование в машиностроении. - 2017. - № 2. - С. 34-38.

14. Перспективы развития технологии и техники горизонтальнонаправленного бурения пилотных скважин для бестраншейной прокладки трубопроводов / Л.А. Саруев, А.В. Шадрина, А.Л. Саруев, С.С. Васенин, А.В. Пахарев // Известия Томского политехнического университета. Инжиниринг георесурсов. 2019. - T. 330. - № 4. - C. 89-97.

15. Kordubailo A.O., Simonov B.F. Downhole periodic electromagnetic seismic source designs // Journal of Mining Science. - 2020. - V. 56. - № 5. - P. 146-153.

16. Ударный узел установки ударно-вращательного бурения: пат. № 79924 Российская Федерация. МПК Е2В 6/04 (2006.01); заявл. 21.07.2008; опубл. 20.01.2009. - Бюл. № 2. - 4 с.

17. Ударный узел станка ударно-вращательного бурения: пат. № 79923 Российская Федерация. МПК Е21В 6/04 (2006.01); заявл. 21.07.2008; опубл. 20.01.2009. - Бюл. № 2. - 4 c.

18. Сердечный А.С. Управление амплитудой и длительностью ударного импульса: автореф. дис. ... д-ра техн. наук. - Новосибирск, 1997. - $31 \mathrm{c}$.

19. Мигулин В.В. Основы теории колебаний. - М.: Наука, 1978. $392 \mathrm{c}$.

20. Еремьянц В.Э., Мелис кызы Назира. Модель ударной системы с жидкостной камерой для разрушения крепких горных пород // Фундаментальные основы механики. - 2017. - № 2. - С. 49-53.

Поступила 24.11.2021 2.

\section{Информация об авторах}

Саруев Л.А., доктор технических наук, профессор отделения нефтегазового дела Инженерной школы природных ресурсов Национального исследовательского Томского политехнического университета.

Шадрина A.B., доктор технических наук, доцент отделения нефтегазового дела Инженерной школы природных ресурсов Национального исследовательского Томского политехнического университета.

Мельнов К.B., аспирант отделения нефтегазового дела Инженерной школы природных ресурсов Национального исследовательского Томского политехнического университета.

Саруев А.Л., кандидат технических наук, доцент отделения нефтегазового дела Инженерной школы природных ресурсов Национального исследовательского Томского политехнического университета. 
UDC 622.233 .63

\title{
RESULTS OF EXPERIMENTAL STUDIES OF THE HYDRAUUC PULSE MECHANSM FOR DRIUNG PILOT WELS DURING PIPEUNE LAYING
}

\author{
Lev A Saruev', \\ saruevla@tpu.ru
}

\author{
Anastasiya V. Shadrina', \\ avshadrina@rambler.ru
}

\author{
Kirill V. Melnov', \\ kvm11@tpu.ru \\ Alexey L. Saruev', \\ saruev@tpu.ru \\ 1 National Research Tomsk Polytechnic University,
30, Lenin avenue, Tomsk, 634050, Russia.
}

The relevance. Hydraulic percussive mechanisms are widely used in mining and other industries. They are currently being actively improved. In particular, numerous publications argue that it is possible to control the amplitude and duration of pressure pulses generated by hydromechanical systems in closed-loop system, through which the plunger transmit the impact energy to the rock-breaking tool. At the same time, increased duration of the power pulses lead to significant increase in the efficiency of rock failure, the excessive dynamic loads on the elements of the drilling tool are reduced. Typically percussion mechanisms have hammer the reciprocating motion of which leads to energy losses. A fundamentally new hammerless hydraulic mechanism was developed. It allows automatic regulating the amplitude and duration of the force pulses it generates, depending on the hardness of the destroyed rock. Nevertheless, it is necessary to conduct special studies in order to substantiate interrelations among its main parameters and operability in general.

The main aim of the research is substantiation and experimental verification of the physical model of new hydraulic percussive mechanism in laboratory settings.

Object of study is new hydraulic percussive mechanism with a closed chamber, where force pulses are formed, making the rock destruction more efficient.

Subject: regularities and relationships of the main kinematic and dynamic parameters of the physical model of the hydraulic percussive mechanism.

Methods: analysis of scientific and technical information regarding field of knowledge; modeling the dynamic processes in hydraulic percussive mechanism and laboratory investigation.

Results. The efficiency of the hammerless hydraulic mechanisms is assessed; its advantages are revealed in comparison with the existing hydraulic percussion mechanisms; it has been established that the hammerless hydraulic mechanisms automatically regulate the amplitude and duration of power pulses depending on the hardness of the destroyed rock and do not require an additional control system for this process; the efficiency of the hydraulic percussive mechanism is higher than in existing hydro percussive systems as it forms power pulses that practically have no high-frequency components, not conducive to the rocks destruction.

\section{Key words:}

Power pulse, high pressure hose, hammerless hydraulic mechanisms, closed-loop system, pressure pulse, pulse amplitude and duration, trenchless pipeline construction.

\section{REFRANCES}

1. Aleksandrova N.I., Kondratenko A.S. Movement of an open-ended pipe with a soil plug under a longitudinal impact. Geotechnical and Geological Engineering, 2020, vol. 38, no. 4, pp. 3493-3504.

2. Kamel M.A., Eishafei M., Saif A.-W.A., Al-Majed, A.A. Automatic trenchless horizontal directional drilling using quad motors drilling heads. Proc. of 15th International Multi-Conference on Systems, Signals and Devices. Tunisia-Hammamet, 2018. pp. 1172-1177.

3. Rabiei M., Chang K.P., Gelinas M., Neale A. Analysis and design of pipes installed via direct PIPE technology. Proc. of Conference: NASTT No-Dig Show. Palm Springs, CA, March 25-29, 2018. $11 \mathrm{p}$.

4. Smolyanitskii B.N., Tishenko I.V., Chervov V.V. Improvement prospects for air hammers in building and construction work. Journal of Mining Science, 2009, vol. 45, no. 4, pp. 363-371.

5. Zhao Z., Meng Y., Li Y., Shi X., Xiang C. Effects of working angle on pneumatic down-the-hole hammer drilling. Rock mechanics and rock engineering, 2014, vol. 48, Iss. 5, pp. 2141-2155.
6. Gorodilov L.V. Basic properties of one-way action hydraulic percussion system with two piston arresters. Journal of mining science, 2020, vol. 56, no. 6, pp. 105-115.

7. Efimov V.P., Gorodilov L.V. Experimental investigation of shock pulses in the piston-bit system in interaction with rock mass. IOP Conf. Series: Earth and Environmental Science, 2021, vol. 773, Available at: https://www.scopus.com/inward/record.uri?eid=2s2.0-85107080391\&doi=10.1088\%2f1755-1315\%2f773\%2f1\% 2f012041\&partnerID= (accessed 15 September 2021).

8. Yonga G., Deshunb L., Shuyib Ya., Xuejuna L., Jianwenc Ch. Hydraulic-mechanical coupling modeling by bond graph for impact system of a high frequency rock drill drifter with sleele distributor. Automation and construction, 2016, vol. 63, pp. 88-99.

9. Tishhenko I.V., Chervov V.V., Smoljanickij B.N. Evaluation of layout of air drill hammer with smooth adjustment of impact impulse frequency. Journal of Mining Science, 2017, vol. 53, no. 1, pp. 110-118.

10. Timonin V.V., Alekseev S.E., Karpov V.N., Chernienkov E.M. Influence of DHT hammer impact energy on drilling-with-casing system performance. Journal of Mining Science, 2018, vol. 54, no. 1 , pp. $61-71$. 
11. Zhukov I.A., Smoljanickij B.N., Timonin V.V. Improvement of down-the-hole air hammer efficiency by optimizing shapes of collding parts. Journal of Mining Science, 2018, vol. 54, no. 2, pp. 37-43.

12. Eremyants V.E., Melis kyzy Nazira. About the collision of the rods model choice through the closed liquid volume. Journal of Advanced Research in Technical Science, 2017, Iss. 6, pp. 11-16.

13. Eremyanc V.E., Melis kyzy Nazira. Model udarnoy sistemy s zhidkostnoy kameroy dlya razrusheniya prochnykh i merzlykh gruntov [Model of a percussion system with a liquid chamber for the destruction of solid and frozen soils]. Avtomatizirovannoe proektirovanie v mashinostroenii, 2017. vol. 2, pp. 34-38.

14. Saruev L.A., Shadrina A.V., Saruev A.L., Vasenin S.S., Pakharev A.V. Prospects for development of technology and facilities of pilot bores horizontal directional drilling for trenchless laying of pipelines. Bulletin of the Tomsk Polytechnic University. Geo Assets Engineering, 2019, vol. 330, no. 4, pp. 89-97. In Rus.

15. Kordubailo A.O., Simonov B.F. Downhole periodic electromagnetic seismic source designs. Journal of Mining Science, 2020, vol. 56, no. 8, pp. 146-153.

\section{Information about the authors}

Lev A. Saruev, Dr. Sc., professor, National Research Tomsk Polytechnic University.

Anastasiya V. Shadrina, Dr. Sc., associate professor, National Research Tomsk Polytechnic University.

Kirill V. Melnov, post-graduate student, National Research Tomsk Polytechnic University.

Aleksey L. Saruev, Cand Sc., associate professor, National Research Tomsk Polytechnic University.
16. Kazantsev A.A., Shadrina A.V., Saruev L.A., Saruev A.L. Udarny uzel ustanovki udarno-vrashchatelnogo bureniya [Percussive mechanism of percussion rotary drilling]. Patent RF no. 79924, 2009.

17. Kazantsev A.A., Shadrina A.V., Saruev L.A., Saruev A.L. Udarny uzel stanka udarno-vrashchatelnogo bureniya [Percussive mechanism of percussion rotary drilling]. Patent RF no. 79924, 2009.

18. Serdechny A.S. Upravlenie amplitudoy $i$ dlitelnostyu udarnogo impulsa. Aftoreferat Diss. Dokt. nauk [The control of pulse height and duration. Dr. Diss. Abstract]. Novosibirsk, 1997. 31 p.

19. Migulin V.V. Osnovy teorii kolebaniy [Fundamentals of vibration theory]. Moscow, Nauka Publ., 1978. 392 p.

20. Eremiants V.E., Melis kyzy Nazira. Model udarnoy sistemy s zhidkostnoy kameroy dlya razrusheniya krepkikh gornykh porod [Model of a percussion system with a liquid chamber for the destruction of hard rock]. Fundamentalnye osnovy mekhaniki, 2017, no. 2, pp. 49.

Received: 24 November 2021. 